Date of Submission:

Email: maiti@physics.iisc.ernet.in

\section{Date of Acceptance:}

\title{
Understanding DNA based Nanostructures
}

\author{
Prabal K. Maiti ${ }^{1+}$, Tod A.Pascal ${ }^{2}$, Nagarajan Vaidehi ${ }^{2,3}$, and William A. Goddard $\mathrm{III}^{2, *}$ \\ ${ }^{1}$ Center for condensed Matter Theory, Department of Physics, \\ Indian Institute of Science, Bangalore, India 560012 \\ ${ }^{2}$ Materials and Process Simulation Center (MSC), (MC 139-74), \\ California Institute of Technology, Pasadena, CA 91125
}

\begin{abstract}
We use molecular dynamics (MD) simulations to understand the structure, and stability of various Paranemic crossover (PX) DNA molecules and their topoisomer JX molecules, synthesized recently by Seeman and coworkers at New York University (NYU). Our studies include all atoms (4432 to 6215) of the PX structures with an explicit description of solvent and ions (for a total of up to 42,000 atoms) with periodic boundary conditions. We report the effect of divalent counterions $\mathrm{Mg}(+2)$ on the structural and thermodynamic properties of these molecules and compare them to our previously reported results in presence of monovalent $\mathrm{Na}+$ ions. The dynamic structures averaged over the 3-nanosecond simulations preserves the WatsonCrick hydrogen bonding as well as the helical structure. We find that PX65 is the most stable structure both in $\mathrm{Na}+$ and $\mathrm{Mg}(+2)$ in accordance with the experimental results. PX65 has helical twist and other helical structural parameters close to the values for normal B-DNA of similar length and sequence. Our strain energy calculations demonstrate that stability of the crossover structure increases with the increase in crossover points.
\end{abstract}

\footnotetext{
* Email: ${ }^{+}$maiti@ physics.iisc.ernet.in and ${ }^{*}$ wag@ @ag.caltech.edu

${ }^{3}$ current address: Division of Immunology, Beckman Research Institute of City of Hope, Duarte, CA 91010
} 


\section{$\underline{1.0 \text { Introduction }}$}

Intermolecular interactions of DNA are highly specific and readily programmable through Watson-Crick complementarity: A pairs with T, G pairs with C. This complementarity can be used to design systems in which single strands self-assemble into double strands, branched junctions, and other more complex motifs. The Seeman laboratory at New York University has exploited specificity of Watson-Crick pairing to synthesize a variety of branched DNA motifs that they have used to self-assemble both nanomechanical device and novel DNA nanostructures such as the cube and the truncated octahedron. Paranemic crossover (PX) DNA molecules and their topoisomer JX molecules, recently synthesized by Seeman and co-workers

${ }^{1,2}$, are emerging as very important building blocks for building nanomechanical devices and for creating self-assembled DNA nanostructures ${ }^{2,3}$. However practical design and manufacture of nanoscale machines and devices requires overcoming numerous formidable hurdles in: synthesis, processing, characterization, design, optimization, and fabrication of the nanocomponents. Each area presents significant challenges to the experimentalist because the properties of nanoscale systems may differ significantly from macroscopic and molecular systems and because the manipulation and characterization of structures at the nanoscale is difficult. Even so, branched motifs of DNA that provide components for the self-assembly of 2D and 3D arrays at the nanoscale have been synthesized. The concepts of crossover points that connect one double helical strand to a strand in a second double helix have been demonstrated to yield rigid motifs of DNA. Thus these crossover points connect two very flexible double helical structures to form a single rigid structure. Such rigid units as the DAO and DAE motif double crossover (DX) molecules ${ }^{4}$ are critical in making a nanomechanical device.

Recently, the Seeman group discovered a new DNA motif, paranemic crossover (PX) DNA and one of its variants, $\mathrm{JX}_{2}$ DNA, that provides the basis for a robust sequence-dependent nanomechanical device ${ }^{1}$. Using the sequence dependence of this device an array of such devices could be organized so that each device would respond individually to a unique set of signals. The crossover points in PX structures occur at every point at which the two strands from each double helix come together, as shown in Figure 1. As illustrated here the PX motifs can be built with varying number of nucleotides in the major and minor grooves. For example PX55 is the PX structure with five nucleotides in the major groove and five in the minor groove. Thus there are 10 nucleotides in one turn of the double helix. Various PX structures such as PX55, 
PX65, PX75, PX85 and PX95 have been synthesized in solution. These PX structures consist of four individual strands that are designed to complement in only one way. The $\mathrm{W}$ and $\mathrm{N}$ notations in the center of the molecule indicate the wide and narrow groove juxtapositions of the two helices.

Sometimes such experiments fail to make the desired structures while often they work as intended, but there is no clear cut understanding of why certain sequences and structures form stable conformations while others do not. Because of this difficulty in experimentally controlling nanostructures and in measuring the properties of nanosystems, it is possible for modeling and simulation to play an essential role in nanoscale synthesis and design. Earlier ${ }^{5,6}$ we have reported the atomistic level structural properties of the PX and JX structure in the presence water and monovalent $\mathrm{Na}+$ ions and demonstrated how simulation can elucidate the structure-property relationships in such DNA nanostructure. In particular we have developed a strain energy analysis method based on the nearest-neighbor interaction and computed the strain energy for the PX molecules compared to the B-DNA molecules of the same length and sequence. We found that PX65 has the lowest calculated strain energy ( - $0.77 \mathrm{kcal} / \mathrm{mol} / \mathrm{bp})$, and the strain increases dramatically for PX75, PX85 and PX95. PX55 has the highest strain energy ( 1.85 kcal/mol/bp) making it unstable, which is in accordance with the experimental results. We also found that PX65 has helical twist and other helical structural parameters close to the values for normal BDNA of similar length and sequence. Vibrational mode analysis shows that compared to other PX motifs, PX65 has the smallest population of the low frequency modes which are dominant contributor for the conformational entropy of the PX DNA structures. All these results indicate that PX65 is structurally more stable compared to other PX motifs in agreement with experiments. These results should aid in designing optimized DNA structures for use in nanoscale components and devices.

Thus these previous simulations also helped explain some of the experimentally observed results in such systems. However, there was some uncertainty in our conclusions because the simulations used $\mathrm{Na}+$ based salts for the counter ions, whereas the experiments were carried out using $\mathrm{Mg}(+2)$ based salts. Indeed the experimental studies indicate that the PX structures are more stable and more reproducible when carried out in $\mathrm{Mg}(+2)$ based solutions rather than $\mathrm{Na}^{+}$.

In this paper we extend these computational studies of the structural properties of the PX structures in the presence of divalent $\mathrm{Mg}(+2)$ ions rather than $\mathrm{Na}+$. Here we carried out molecular dynamics simulations in explicit water with $\mathrm{Mg}(+2)$ salt to predict the structural 
properties of the PX structures. This is the first time that such large DNA based nanostructures have been simulated in explicit water for such long time scales. The paper is organized as follows:

The structure building and the simulation methods are presented in section 2. The results from the molecular dynamics simulation on various PX structures are presented in section 3 . Finally, a summary of the main results and the conclusions drawn from these are given in section 4.

\section{$\underline{2.0 \text { Methods }}$}

\subsection{Building and Simulation Details for the PX structures}

The details of the construction procedure and simulation for the PX and JX structures has been published elsewhere ${ }^{5,6}$. Here we outline the basic steps involved. We first constructed two regular B-DNA structures with the base pair sequence shown in Figure $\mathbf{1}$ and accommodated different number of base pairs per helical turn by adjusting the twist angle of a selected number of base pairs. The individual double helices were built using Namot2 (version 2.2.) ${ }^{7}$. The two double helices thus built in Namot2, were oriented so that the 5' and 3' ends of the double helices are parallel to the $y$-axis and the individual helices rotated so that the desired crossover points are at the closest distance to each other. Once we had identified the suitable crossover points, we created the crossovers using the "nick" and "link" commands in Namot2. These structures were saved in the Protein Database (PDB) file format. Figure 2 shows the snapshot of the built PX structures after minimizations.

\subsection{Simulation Details for the PX - JX structures}

All MD simulations reported in this paper used the AMBER7 software package ${ }^{8}$ with the all-atom AMBER95 force field $(\mathrm{FF})^{9}$. For $\mathrm{Mg}(+2)$ we used the Aqvist ${ }^{10}$ interaction parameters. The electrostatics interactions were calculated with the Particle Mesh Ewald (PME) method ${ }^{11,12}$ using a cubic B-spline interpolation of order 4 and a $10^{-4}$ tolerance set for the direct space sum cutoff. A real space cut off of $9 \AA$ was used both for the electrostatics and van-der Waals interactions with a non-bond list update frequency of 10.

Using the LEAP module in AMBER, the PX/JX nanostructures were immersed in a water box using the TIP3P model for water. The box dimensions were chosen in order to ensure a $10 \AA$ solvation shell around the DNA structure. In addition, some waters were replaced by $\operatorname{Mg}(+2)$ counter ions to neutralize the negative charge on the phosphate groups of the backbone of the $\mathrm{PX} / \mathrm{JX}$ structures. This procedure resulted in solvated structures, containing approximately 
42,000 atoms in a box of dimensions $45 \AA$ x $65 \AA$ x $196 \AA$. These PX and JX structures were subjected to the equilibration protocol outline in our previous work ${ }^{5,6}$.

\subsection{Results and Discussion}

3.1 Differences in Flexibility of the PX structures

$\mathrm{MD}$ simulations in the presence of monovalent $\mathrm{Na}+$ ions and divalent $\mathrm{Mg}(+2)$ ions have been reported previously for the crystal structure of B-DNA to validate the AMBER force field (FF ${ }^{13}$, also using explicit salt and water and the particle mesh Ewald method for calculating the non-bond electrostatic interactions ${ }^{14-17}$. The simulations on crystalline B-DNA led to an overall calculated CRMSD for all atoms of 1.0-1.5 $\AA^{14-17}$. For the solution phase, there are no reliable experimental structures with which to compare the simulations, which generally lead to RMSD differences of 3.6-4.2 $\AA$ from the crystal ${ }^{16,17}$. However, the effect of $\mathrm{Mg}(+2)$ ions on the structure of DNA in water has not been reported. A few simulations have been reported on the influence of $\mathrm{Mg}(+2)$ ions on the DNA structure ${ }^{17,18}$. Our present simulation study also helps us to understand the binding of $\mathrm{Mg}(+2)$ ions to DNA.

We carried out MD simulations for 2.5 to 3 ns in explicit salt and water for each of the five PX nanostructures (PX55, PX65, PX75, PX85, and PX95) at 300K. In each case we define an average MD structure by averaging the coordinates for various snapshots for the last $1 \mathrm{~ns}$ at an interval of $1 \mathrm{ps}$. This structure represents the time averaged solution structure of the PX nanostructures (that one would compare to an NMR structure). These averaged structures for various PX structures are shown in Figure 3(a) (in presence of $\mathrm{Na}^{+}$) and 3(b) (in presence of $\operatorname{Mg}(+2))$.

In the presence of monovalent $\mathrm{Na}+$ ions the base stacking and Watson-Crick hydrogen bonding are well maintained in the solution structure for all the PX molecules. However, the PX55, PX85, and PX95 structures undergo substantial writhing (as can be seen from the side view of the PX structures), whereas PX65 and PX75 do not. Based on this feature PX65 and PX75 should be most suitable for creating 2-D arrays of nanostructures.

We also find that the presence of divalent $\mathrm{Mg}(+2)$ ions leads to significant changes in the solution properties of PX molecules compared to $\mathrm{Na}+$ containing solutions. Thus $\mathrm{Mg}(+2)$ ions lead to a significantly distortion in the Watson-Crick hydrogen bonding among the bases and also to large bending of the double helix for PX95. Further quantitative insight into the base pairing and other structural features is obtained from the calculated helical parameters shown in section 3.2. 
To obtain some measure of the flexibility of these structures, Figure 4(a) and (b) show the CRMSD of the instantaneous PX snapshots from the time averaged solution structure as a function of time. This CRMSD was also calculated for the entire 3 ns MD runs, representing the fluctuations of a PX structure in solution. We see that PX55 and PX65 have the least fluctuations in solution with an average CRMSD of 2.0-2.5 $\AA$ over the final $1 \mathrm{~ns}$ both in the presence of $\mathrm{Na}+$ as well as Mg(+2) ions. The other three cases, PX75, PX85 and PX95, have larger fluctuations of 2.5-3.0 $\AA$, revealing a lower structural ordering in solution.

The CRMSD with respect to the initial minimized canonical structure is shown in Figure 5(a) for all the PX structures. The root-mean-square deviation in coordinates (CRMSD) from the initial canonical structure remains within 3-4 $\AA$ for PX65 over the dynamics, but it goes up to 8-12 $\AA$ for PX55, PX75, PX85 and PX95. For comparison we show the CRMSD of the PX structure in presence of $\mathrm{Na}+$ ions in figure 5(b). Here again CRMSD from the initial canonical structure remains within 3-4 $\AA$ for PX65 over the dynamics, but goes up to 7-8 $\AA$ the other PX structures. This indicates the intrinsic stability of PX65 structure compared to other PX structures, which agrees with the experimental results.

\subsection{Comparison of the helicoidal parameters for the PX nanostructures}

More critical structural characterization of the PX/JX DNA nanostructure can be made by calculating various helicoidal structural parameters such as roll, rise, tilt, and twist. These parameters were calculated for each base pair, averaged over last 400 ps of the 3 ns dynamics run. The Curve algorithm ${ }^{19,20}$ was used to calculate the various helicoidal parameters.

Divalent cations like $\mathrm{Mg}(+2)$ are known to have sequence specific binding to the DNA and to affect the major/minor groove binding properties ${ }^{21}$. They are also known to produce significant bending of the double helical geometry. Figures 6 shows the rise, tilt, roll, and twist calculated for every base pair for the PX65 structure in the presence of $\mathrm{Mg}(+2)$ ions. The helical twist angle for the two double helices of the PX65 fluctuates around $31^{\circ}$ and the base tilt angle fluctuates around $0.31^{\circ}$ (essentially zero). These values are close to the values ( $30^{\circ}$ for twist and $0.22^{\circ}$ for tilt) obtained from simulation of the two separated double helices of PX65 ${ }^{5}$. Thus the helical parameters for PX65 are close to that of B-DNA double helix and hence PX65 should be a very stable structure like a B-DNA. On the other hand, for the PX55, PX75, PX85 and PX95 
structures the helical twist and tilt angles show large fluctuations about the corresponding values in B-DNA. These fluctuations are especially large at the crossover points. The presence of $\mathrm{Mg}(+2)$ has resulted in significant distortion of the PX95 structure as is evident from various helicoidal parameters.

Figures 7(a) and 7(b) show the variation of the width of the major and minor grooves in each of the two double helices for PX65 in presence of $\mathrm{Na}+$ and $\mathrm{Mg}(+2)$ respectively.. The major and minor grooves show a steady increase in the width in going from the PX55 to the PX95 structure. The average major groove width for a B-DNA is around $11.7 \AA$, which can widen to $15 \AA$ on binding a protein or drug ${ }^{22}$. The minor groove width in B-DNA is $5.7 \AA$. The PX55 structure shows average width of $12.9 \AA$ for major groove and $5.5 \AA$ for minor groove, which is close to the values for B-DNA. These values increase for PX65 [13.9 $\AA$ for major groove and 6.4 $\AA$ for minor groove] eventually going up to $15-17 \AA$ for the major groove of PX95. The instantaneous major groove width deviates significantly from the average value for each nucleotide, especially at the crossover points. We see the decrease in the major groove width in presence of $\mathrm{Mg}(+2)$ ions.

\subsection{Macroscopic structural properties of PX nanostructures}

We calculated the macroscopic structural features such as writhing, overall bending, and the solvent accessible surface area of the PX structures. We also analyzed the vibrational modes of the PX structures to understand the relation between the low frequency modes and structural stability. These properties throw light on the nature of the PX nanostructures.

\subsubsection{Writhing in longer PX DNA nanostructures}

Figure 8 shows the variation of "strand shortening" for various PX structures averaged over the last 200 ps of the 3 ns MD simulation runs. Strand shortening is calculated as follows: The Curve algorithm outputs the vectorial direction of each local helical axis segment $U$ and its reference point $P$. The path length between successive helical axis reference points can be calculated as

$$
\text { path }=\sum_{i}\left|\begin{array}{c}
\vec{P}_{i}-\vec{P}_{i-1}
\end{array}\right|
$$

and the end-to-end distance of the DNA fragment can be calculated as 


$$
R_{e}=\left|\vec{P}_{1}-\vec{P}_{N}\right|
$$

where $\vec{P}_{1}$ and $\vec{P}_{N}$ are the reference points for the two end helical axis corresponding to two terminal nucleotides. The difference between sum of all the path lengths and the end-to-end distance is a measure of the strand shortening. The strand shortening also indicates the overall flexibility of the DNA.

Figure 8 shows that the end-to-end distance decreases or the strand shortens more as the number of base pairs increase in the PX structures, indicating that strand shortening is highest for PX95. In the presence of $\mathrm{Mg}(+2)$ strands shorten much stronger than in the presence of $\mathrm{Na}+$ ions. This could be rationalized by the fact that presence of $\mathrm{Mg}(+2)$ contributes to the overall charge neutralization of the phosphate backbone thereby making the helix more flexible. Also there is substantial distortion (e.g., writhing and/or bending) in the overall PX95 structure. This effect could be due to the total length or to the 95-crossover motif that places 14 base pairs in one helical turn or due to base sequence which is intrinsically unstable.

We have also calculated global helical bending for each of the two helices using the algorithm developed by Strahs and Schlick ${ }^{23}$. This method computes the DNA curvature by summing the projected components of local base pair step tilt and roll angles after adjusting the helical twist. Our analysis for the global angles is based on the values of local tilt and roll angles for each base pair step computed by the Curves program ${ }^{24}$. Figure 9 shows the global bending for each PX molecule. The curvature of the double helix axis is similar for both helices in the PX55 molecule. On the other hand the curvature of the two helical axes show a bending angle differing by $10-40^{\circ}$ for the PX65, PX75, PX85, and PX95 structures, indicating the effect of writhing of the helical axis for these structures. Combining the effect of strand shortening with the bending, we infer that PX95 shows a larger writhing in its solution structure compared to the other PX structures. The effect of writhing is likely to be an important structural feature in designing nanostructures. For example, because of the writhing PX95 may not be a good choice for constructing a 2-D array using PX nanostructures.

\subsection{Relative Stability of the PX/JX nanostructure}

3.4.1 Relative stability of the JX structures:

The PX DNA is a four-stranded molecule in which two parallel double helices are joined by reciprocal exchange of strands at every point at which the strands come together ${ }^{1,2}$. The JXM structure is related to PX by containing M adjacent sites where backbones of the two parallel 
double helices juxtapose without crossing over. Earlier we used Molecular dynamics simulation to demonstrate ${ }^{5}$ that in the presence of the $\mathrm{Na}+$ ions, JX motifs are not able to maintain the parallel double helix crossover structures. Thus the two helical domains of the crossover structure move increasingly far apart with decreasing number of crossover points. However, experimentally JX molecules are found to form in the presence of $\mathrm{Mg}(+2)$ ions. To test the stability of the JX stricture we have calculated ${ }^{25}$ the relative stability of the JX structure as a function of crossover points in the presence of $\mathrm{Mg}(+2)$ ions and find that $\mathrm{Mg}(+2)$ ion induces an effective attraction between the two helical domains to maintain their crossover structures.

\subsubsection{Solvent accessibility surface of PX structures:}

The thermodynamic stability of the PX/JX nanostructure is also greatly influenced by its interaction with the surrounding solvent medium as well as its interaction with the counterions. The solvent accessible surface area (SASA) gives useful insight into the nature of interaction of the PX motifs with the water. The SASA of the PX structures was calculated using the Analytical volume generalized Born (AVGB) method developed in Goddard group ${ }^{26}$. AVGB is very fast and accurate and has been applied successfully to study solvation effects in biological systems ${ }^{27}$. Figures 10(a) and (b) shows the SASA of the PX molecules in the presence of $\mathrm{Na}(+)$ and $\mathrm{Mg}(+2)$ respectively. In the presence of $\mathrm{Na}(+)$ SASA increases from PX55 to PX75 but decreases for PX85 and then increases further for PX95. This observation is consistent with the number of water molecules at the surface of each PX structure shown in Figures 11 (a)-(b). Figure 11(a) shows that the number of water molecules in the first solvation shell of the PX structures increases steadily from PX55 to PX95 with a break at PX85. This could be caused by local kinks present in the PX85 structure although the overall bending of PX85 is not as high as PX95. The presence of $\mathrm{Mg}(+2)$ apparently reduces the number of water in the vicinity of the DNA as the degree of bending of the helical axis increases compared to case when $\mathrm{Na}(+)$ ions are present.

\subsection{Summary and Conclusions}

We have demonstrated that MD simulations over several ns provide critical information on the structural features and relative stability of the various DNA motifs that provide the building blocks for DNA based nanostructures. Our present study fills the gap in the existing literature to provide a comprehensive understanding of the effect of binding of the $\mathrm{Mg}(+2)$ ions on the DNA structural properties. Our study clearly demonstrates how the binding 
of the $\mathrm{Mg}(+2)$ ions affect the structural properties of DNA nanostructures. Calculated CRMSD values along with various helicoidal parameters suggest that PX65 molecule is the most stable molecule in accordance with the experimental findings. Our strain energy analysis based on the nearest neighbor interactions model shows that the molecule with a higher number of crossovers has a higher thermodynamic stability, but that $\mathrm{Mg}(+2)$ ions help stabilize those JX structures with fewer crossover points. .

\subsection{Acknowledgements}

We thank Prof. Nadrian Seeman (NYU) for useful discussions on this subject and sharing his experimental data with us prior to publication. We also thank Mr. Gene Carter for collaborating with us in making changes to his Namot2 code to facilitate building of PX crossover points. PKM thanks SERC, IISc, Bangalore, for computational facility, where some of the computations have been carried out. This research was funded partially from NSF (NIRT 005-610 and CTS- 0548774).

\section{$\underline{\text { References }}$}

$1 \quad$ H. Yan, X. P. Zhang, Z. Y. Shen, and N. C. Seeman, Nature 415 (6867), 62 (2002).

Z. Y. Shen, H. Yan, T. Wang, and N. C. Seeman, Journal of the American Chemical Society 126 (6), 1666 (2004).

$3 \quad$ N. C. Seeman, Biochemistry 42 (24), 7259 (2003).

$4 \quad$ T. J. Fu and N. C. Seeman, Biochemistry 32 (13), 3211 (1993).

5 P. K. Maiti, T. A. Pascal, N. Vaidehi, and W. A. Goddard III, NUCLEIC ACIDS RES 32 (20), 6047 (2004).

6 P. K. Maiti, T. A. Pascal, N. Vaidehi, J. Heo, and W. A. Goddard III, Biophysical Journal 90, 1463 (2006).

$7 \quad$ C. S. Tung and E. S. Carter, CABIOS 10, 427 (1994).

8 D. A. Case, D. A. Pearlman, J. W. Caldwell, T. E. Cheatham, J. Wang, W. S. Ross, C. Simmerling, T. Darden, K. M. Merz, R. V. Stanton, and e. al., AMBER7 (University of California, San Francisco., 1999).

$9 \quad$ W. D. Cornell, et al., J. Am. Chem. Soc. 117, 5179-5197 (1995).

10 J. Aqvist, Journal of Physical Chemistry B 94, 8021 (1990).

11 T. Darden, D. York, and L. Pedersen, Journal of Chemical Physics 98 (12), 10089 (1993).

12 U. Essmann, L. Perera, M. L. Berkowitz, T. Darden, H. Lee, and L. G. Pedersen, Journal of Chemical Physics 103 (19), 8577 (1995).

13 W. D. Cornell, P. Cieplak, C. I. Bayly, I. R. Gould, K. M. Merz, D. M. Ferguson, D. C. Spellmeyer, T. Fox, J. W. Caldwell, and P. A. Kollman, Journal of the American Chemical Society 117 (19), 5179 (1995).

14 H. Lee, T. Darden, and L. Pedersen, Chemical Physics Letters 243 (3-4), 229 (1995). 
D. M. York, W. T. Yang, H. Lee, T. Darden, and L. G. Pedersen, Journal of the American Chemical Society 117 (17), 5001 (1995).

16 K. Miaskiewicz, J. Miller, M. Cooney, and R. Osman, Journal of the American Chemical Society 118 (38), 9156 (1996).

17 D. R. Bevan, L. P. Li, L. G. Pedersen, and T. A. Darden, Biophysical Journal 78 (2), 668 (2000).

18 A. D. MacKerell, Journal of Physical Chemistry B 101 (4), 646 (1997).

19 R. Lavery and H. Sklenar, J. Biomol. Struct. Dyn. 6, 655 (1989).

20 R. Lavery and H. Sklenar, J. Biomol. Struct. Dyn. 6, 63 (1989).

21 T. K. Chiu and R. E. Dickerson, Journal of Molecular Biology 301 (4), 915 (2000).

22 M. A. Schumacher, M. C. Miller, S. Grkovic, M. H. Brown, R. A. Skurray, and R. G. Brennan, Embo Journal 21 (5), 1210 (2002).

$23 \quad$ D. Strahs and T. Schlick, Journal of Molecular Biology 301 (3), 643 (2000).

$24 \quad$ R. Lavery and H. Sklenar, Journal of Biomolecular Structure \& Dynamics 6 (1), 63 (1988).

25 P. K. Maiti, T. A. Pascal, N. Vaidehi, and W. A.Goddard III, (unpublished) (2006).

26 G. Zamanakos, Phd Thesis, Caltech, 2002.

27 N. Vaidehi, W. B. Floriano, R. Trabanino, S. E. Hall, P. Freddolino, E. J. Choi, G. Zamanakos, and W. A. Goddard, Proceedings of the National Academy of Sciences of the United States of America 99 (20), 12622 (2002). 


\section{Figure Captions}

Figure 1: The base pair sequences used in the generations of PX55, PX65, PX75 PX85, and PX95.

Figure 2: Minimized structure for PX55, PX65, PX75 PX85, and PX95.

Figure 3: Structures for various PX molecules averaged over the last 1 ns of molecular dynamics (a) in presence of $\mathrm{Na}(+)$ and (b) in presence of $\mathrm{Mg}(+2)$. For clarity water molecules and counter ions are not shown.

Figure 4: Variation of the coordinate rms displacement (CRMSD) of all atoms of various snapshots from the MD simulation run with respect to the average dynamics structure (a) in presence of $\mathrm{Na}+$ and (b) in presence of $\mathrm{Mg}(+2)$

Figure 5: : Variation of the coordinate rms displacement (CRMSD) of all atoms of various snapshots from the MD simulation run with respect to the initial minimized structure (a) in presence of $\mathrm{Mg}(+2)$ and $(b)$ in presence of $\mathrm{Na}+$.

Figure 6: Average Rise, Tilt, Roll, and twist for PX65. Solid line is for helix1 and broken line is for helix2. The vertical lines corresponds to the crossover points. The data has been averagred over last 200 ps of the 3ns long dynamics. The horizontal solid lines gives the upper bound and lower bound for the corresponding quantities expexted for the helices in their B-DNA form (noncrossover form) during the dynamics.

Figure 7: Average major groove width and minor groove width for PX65 in presence of (a) $\mathrm{Na}+$ and (b) $M g(+2)$. Solid black and blue lines represent major groove and minor groove width respectively for helix1. Broken black and blue lines is for helix2. The vertical lines correspond to the crossover points. The data have been averagred over last 200 ps of the 3ns long dynamics.

Figure 8: Strand shortening as defined in text for various $P X$ molecules. The data have been averaged over last 200 ps of the dynamics run.

Figure 9: Global bend angle calculated for each double helix of each $P X$ structure. The error bars indicate the fluctuations that occur in the molecular dynamics simulation.

Figure 10: Solvent accessible surface area (SASA) per base pair for various PX molecules. The area has been avergaed over last 200 snapshots of the dynamics. The area has been calculated by a very fast and accurate AVGB alogorithm ${ }^{26}$.

Figure 11: Number of water in the first solvation shell (within a shell of $2 \AA$ ) of the PX molecules in presence of (a) $\mathrm{Na}+$ and (b) $\mathrm{Mg}(+2)$.
Deleted: $I$ do not see the $a$ and $b$. It is bettere to put the labels PX6 and PX95

directly in the figure (upper left). 

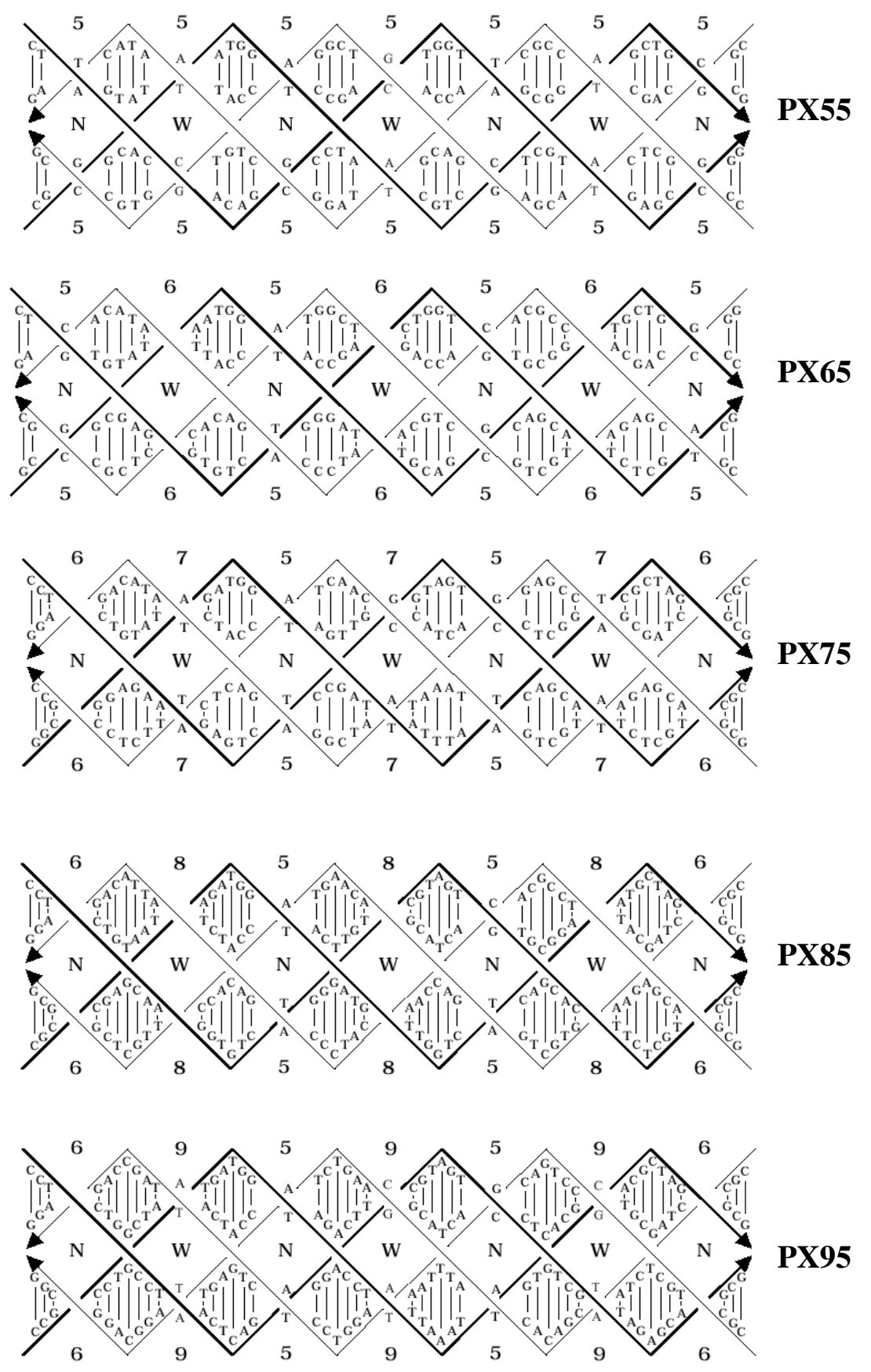

Figure. 1 


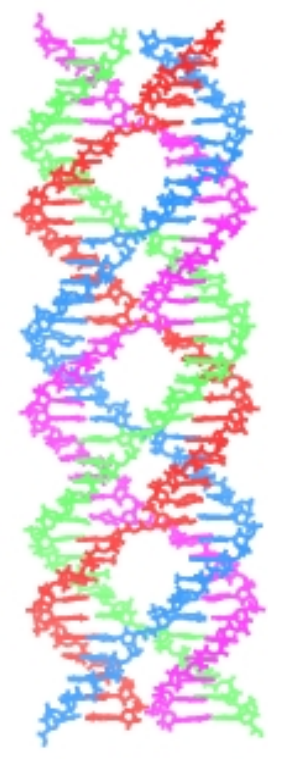

PX 5:5

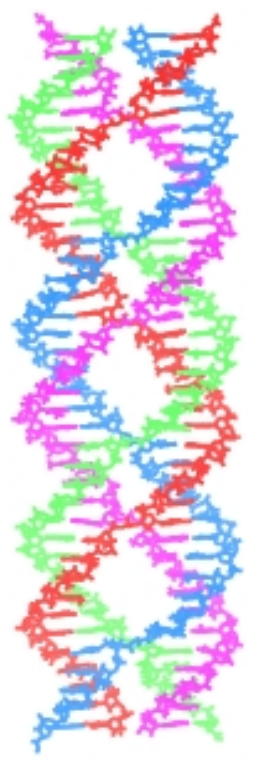

PX 6:5

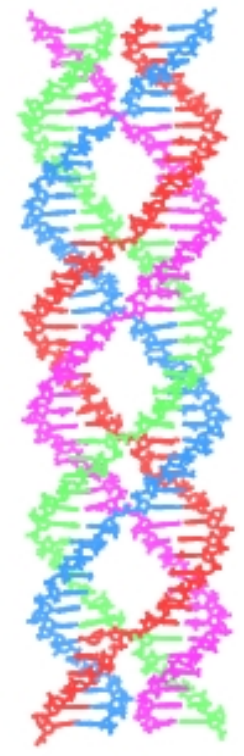

PX 7:5

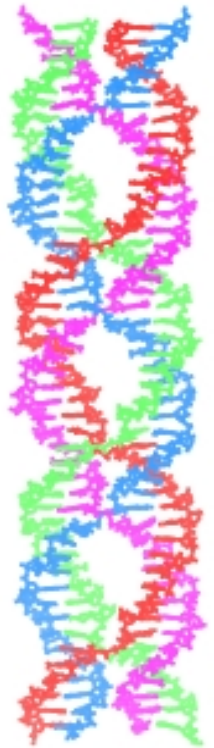

PX 8:5

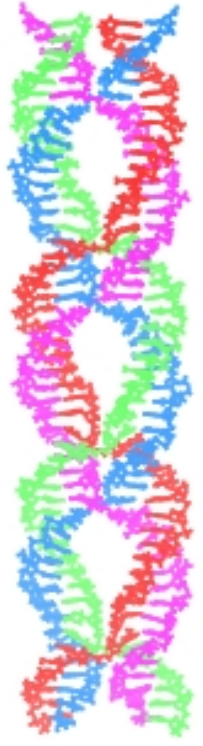

PX 9:5

Figure 2: 

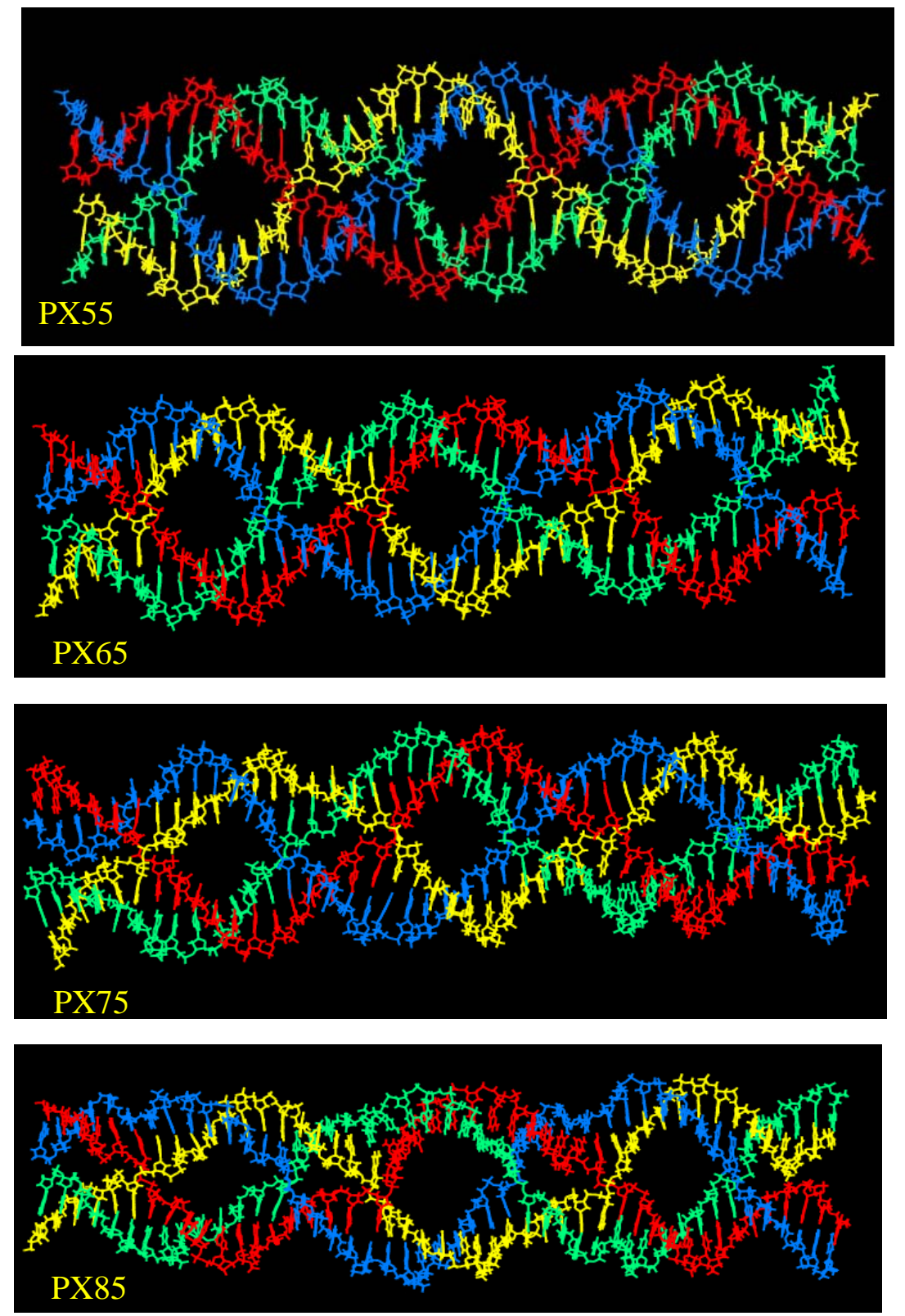


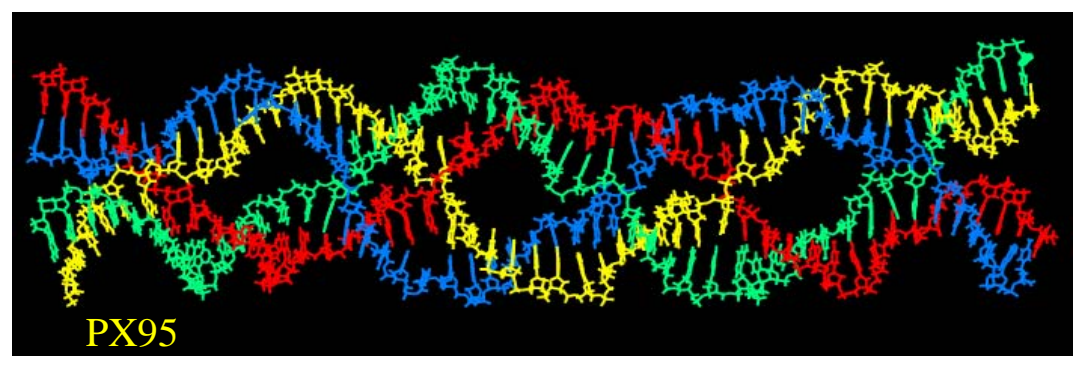

Figure 3 (a). 

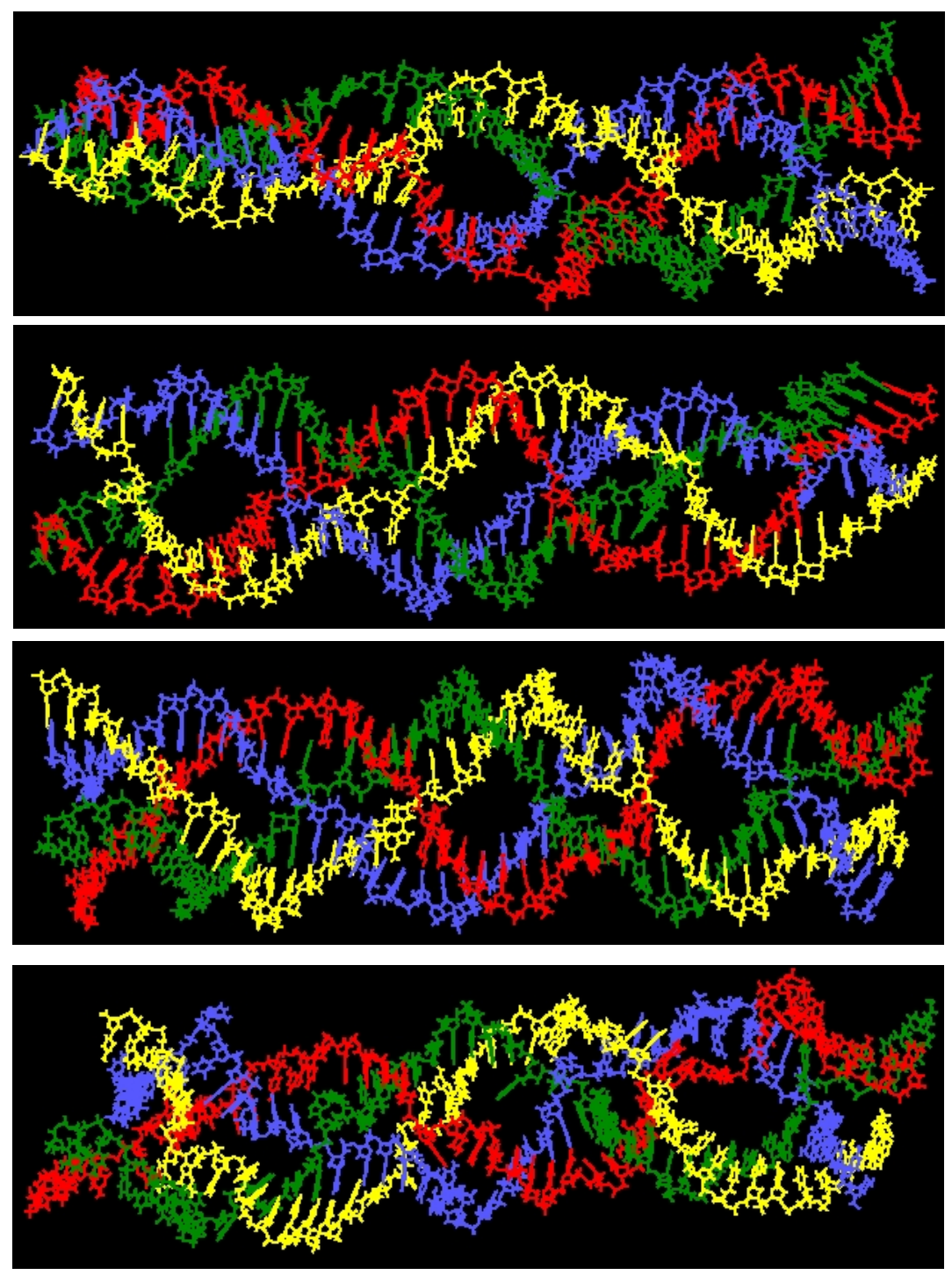


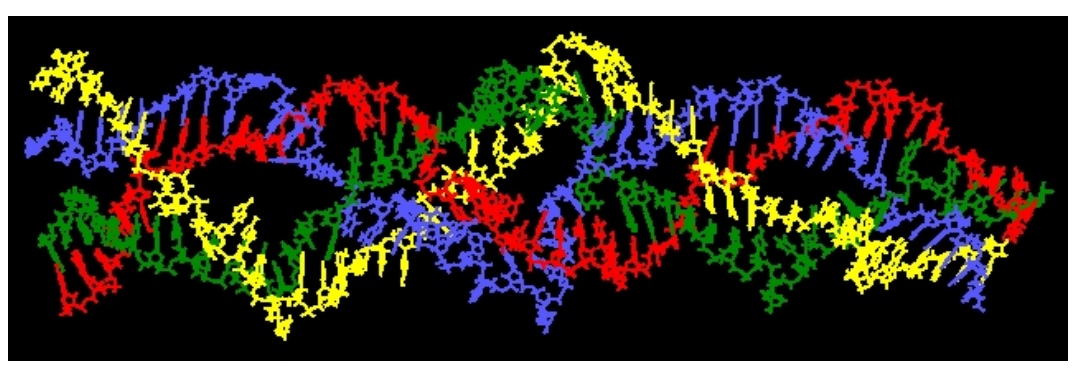

Figure 3(b): 

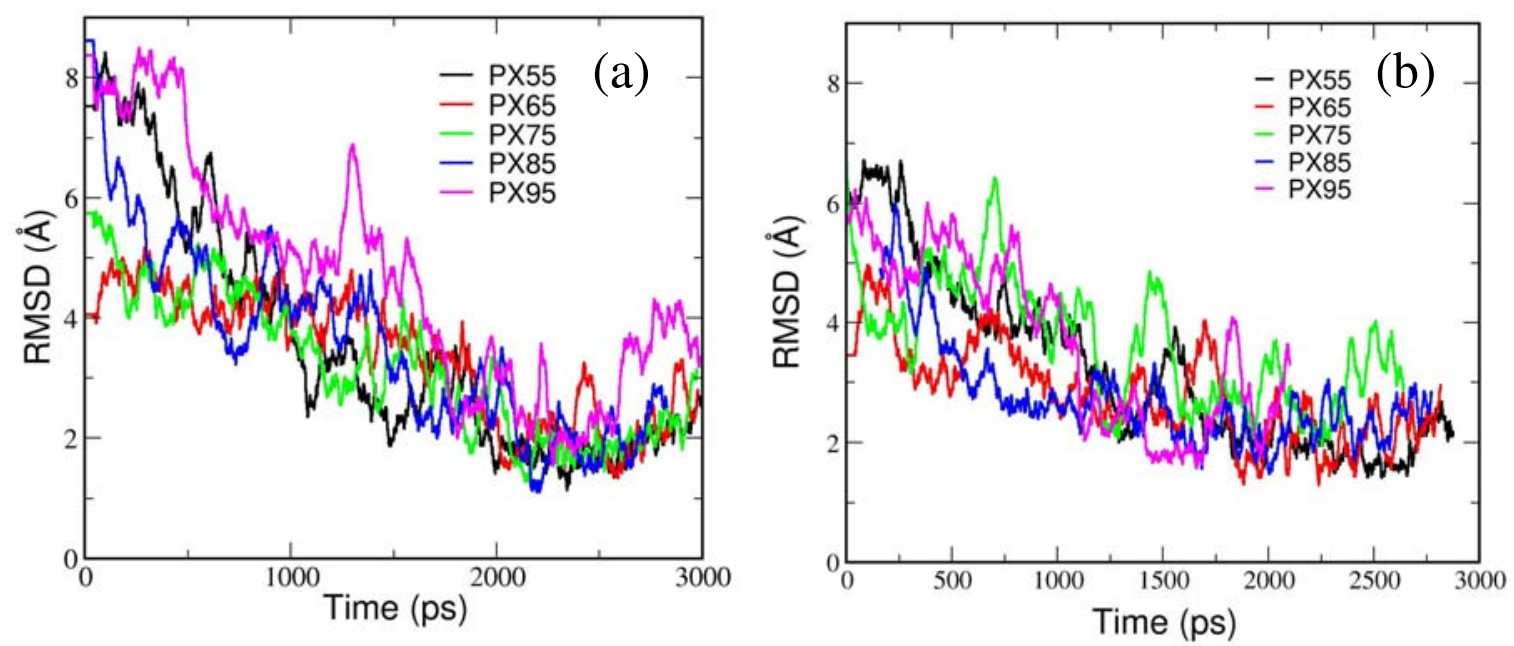

Figure 4 

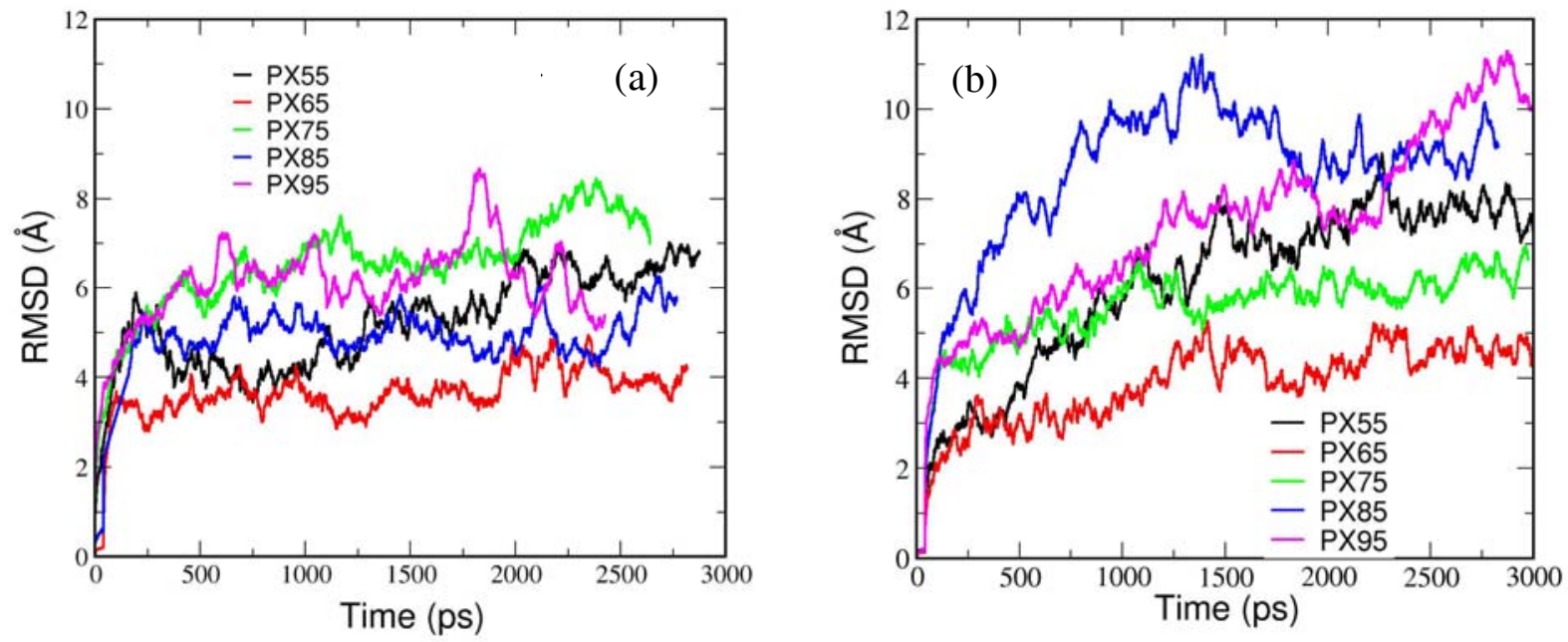

Figure 5. 
$\operatorname{Rise}(\AA ̊)$

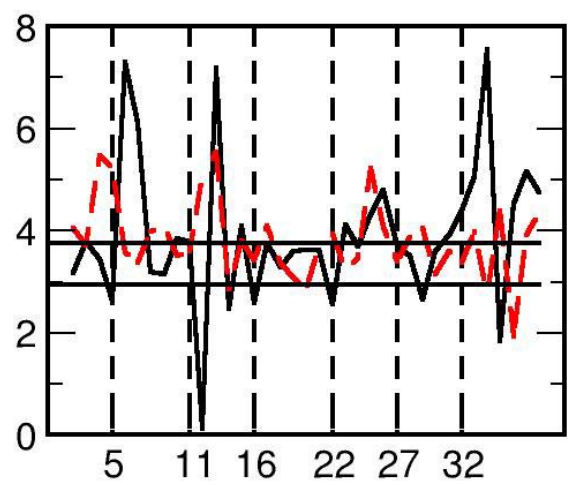

Roll (degrees)

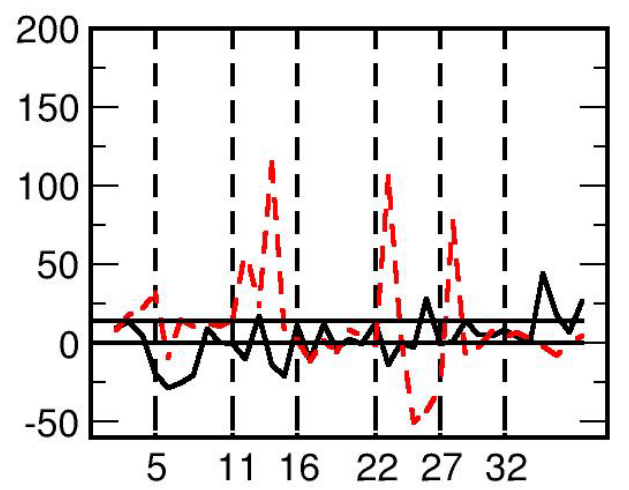

Tilt(degrees)

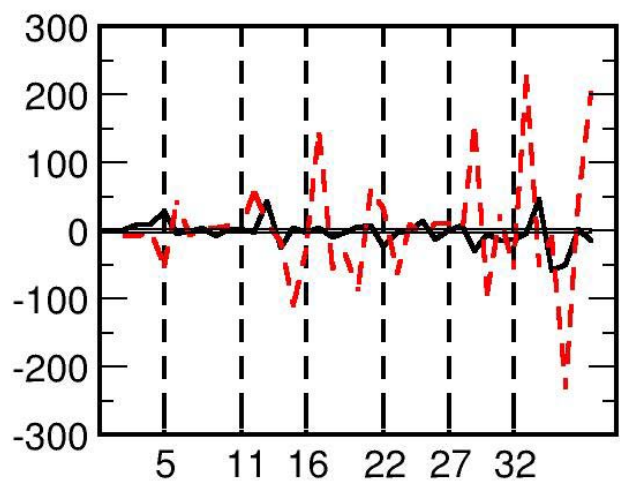

Twist (degrees)

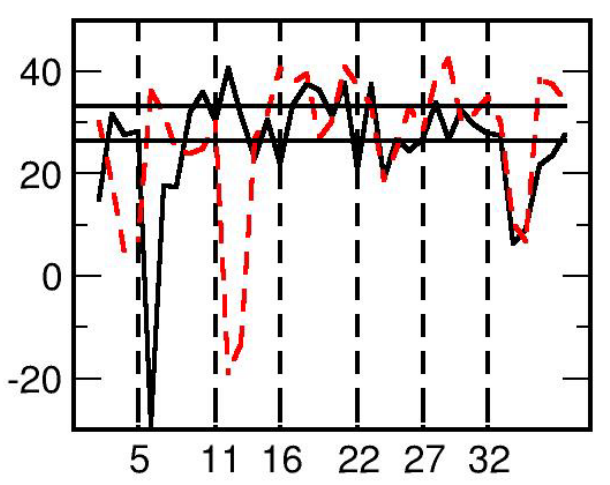

Figure 6 . 

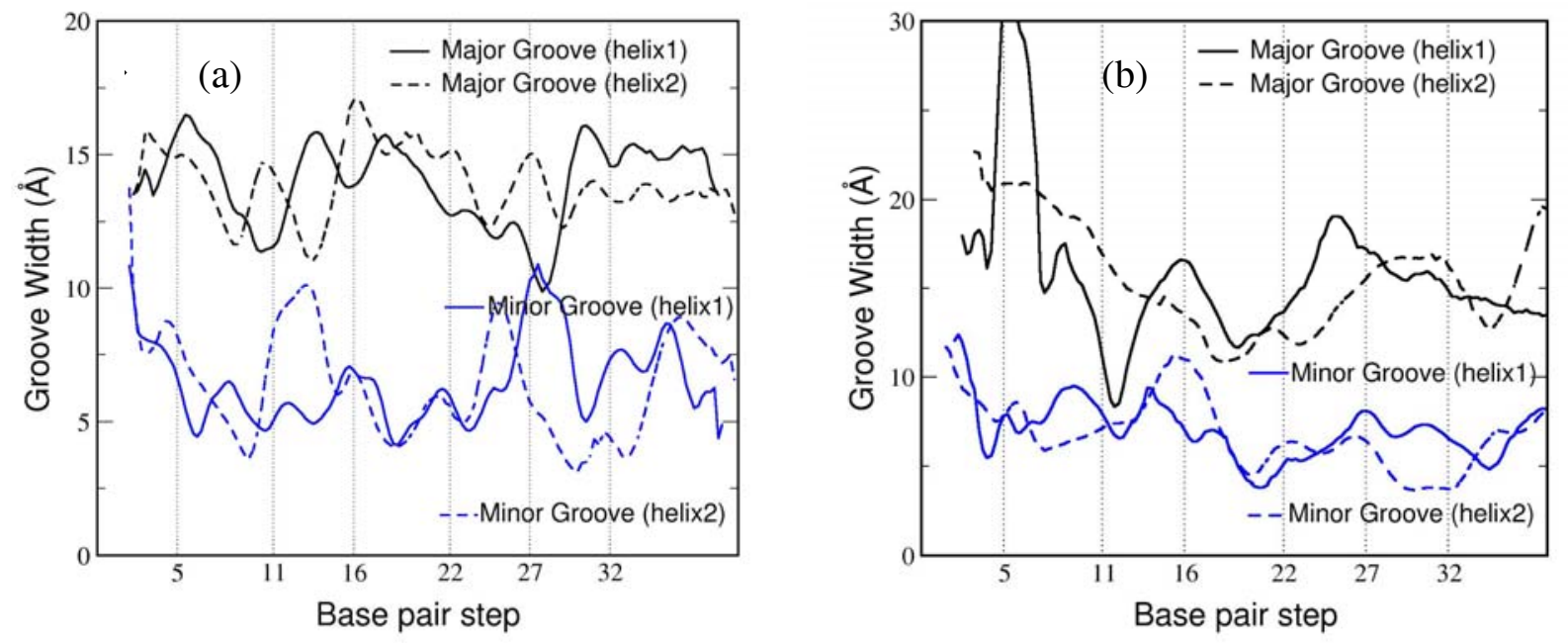

Figure 7. 


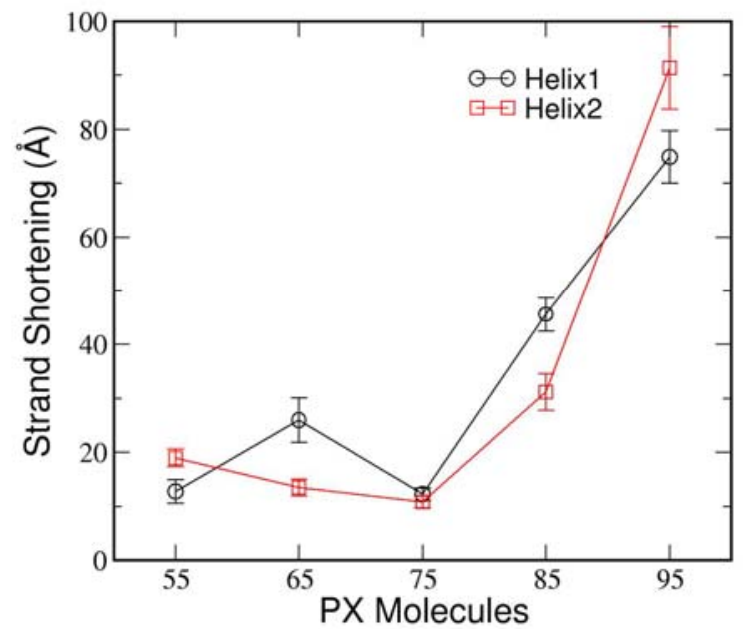

Figure 8 . 


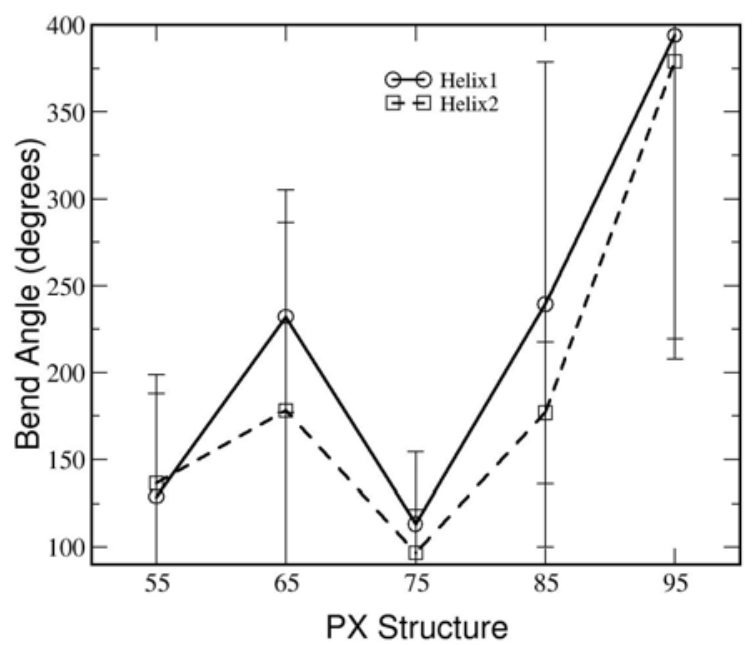

Figure 9. 

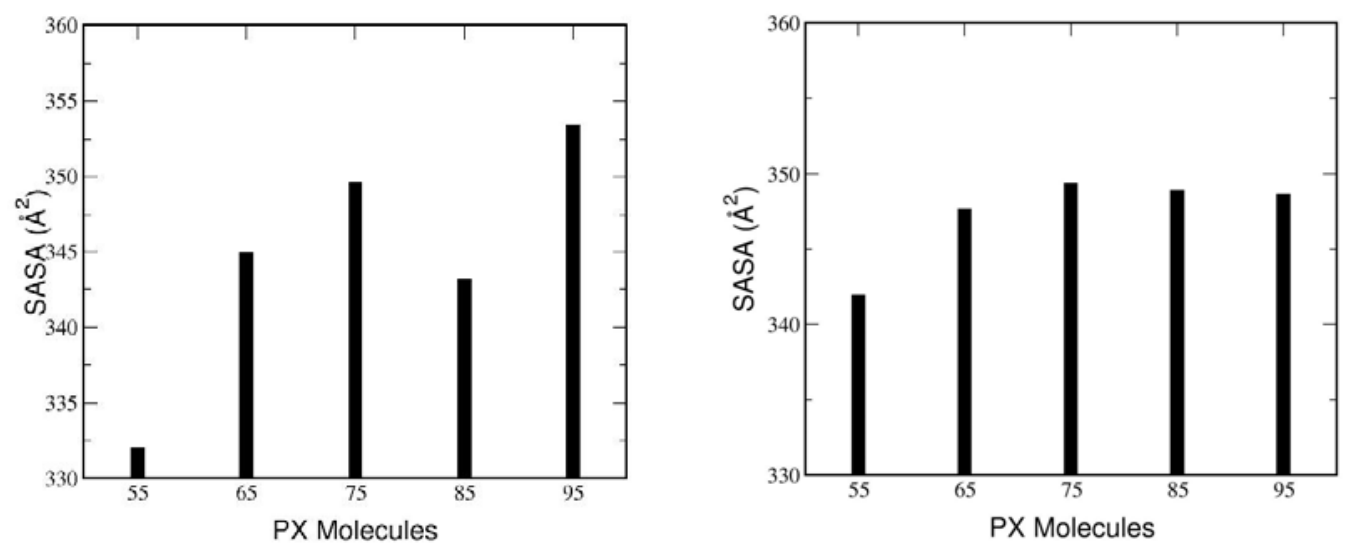

Figure 10 

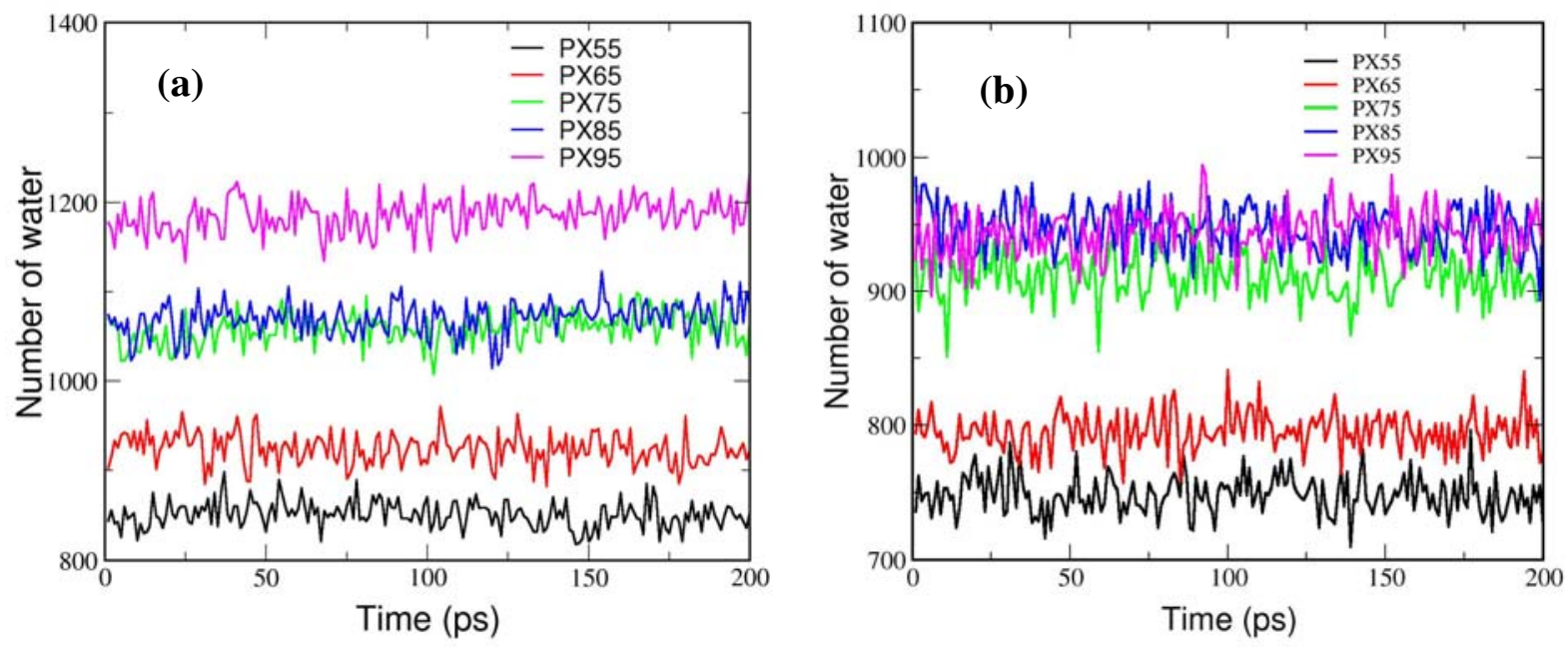

Figure 11: 